\title{
Holographic low concentration optical system increasing light collection efficiency of regular solar panels
}

\author{
Jianbo Zhao ${ }^{\text {a,** Benjamin Chrysler, }}{ }^{\text {b }}$ and Raymond K. Kostuk ${ }^{a, b}$ \\ ${ }^{a}$ University of Arizona, Electrical and Computer Engineering Department, Tucson, \\ Arizona, United States \\ bUniversity of Arizona, James C Wyant College of Optical Sciences, \\ Arizona, United States
}

\begin{abstract}
Conventional photovoltaic (PV) modules (exclude interdigitated back contact modules) with silicon or gallium arsenide PV cells often have significant inactive module surface area. This results from wafer cutting techniques and metal contacts that reduce the module's collection area and the resultant power conversion efficiency. A holographic light collector (HLC) combining a low-cost holographic optical element and a diffuser into conventional PV modules is proposed and evaluated to collect the solar illumination over the inactive module area. The angular tolerance and extra annual energy yield (EY) of the HLC are analyzed. It is found that improvements in EY of 4.5\%, 4.1\%, and 3.8\% can be obtained when PV panels are deployed with two-axis tracking systems, single-axis tracking systems, and without tracking systems, respectively. (C) 2021 Society of Photo-Optical Instrumentation Engineers (SPIE) [DOI: 10 .1117/1.JPE.11.027002]
\end{abstract}

Keywords: photovoltaic module; holographic light collector; holographic optical element; diffuser; waveguide.

Paper 21005 received Jan. 21, 2021; accepted for publication May 6, 2021; published online May 25, 2021.

\section{Introduction}

During the past several decades, photovoltaic (PV) solar energy conversion has played an increasingly important role as a renewable energy source. ${ }^{1}$ The $\$ / \mathrm{W}_{\mathrm{PV}}$ costs of $\mathrm{PV}$ modules have dropped dramatically, primarily due to improved manufacturing methods and increased solar cell efficiency. ${ }^{1,2}$ It has been reported that individual PV cell efficiencies are approaching $27 \%,{ }^{3}$ and the best module power conversion efficiency is about $23 \% .{ }^{4}$ However, the $\$ / \mathrm{W}_{\mathrm{PV}}$ is not expected to decrease hugely due to the maturing wafer manufacturing industry and the small difference between the experimental efficiency and the theoretical efficiency limitation. ${ }^{5}$ Thus in order to increase the energy yield (EY) of a regular PV module, new light collection techniques with low component and manufacturing costs need to be employed. ${ }^{6}$ As efficient, lightweight, and low-cost methods, holographic solar concentration techniques such as holographic lens, planar holographic concentrator, and spectrum splitting have shown significant potential in increasing the EY of PV systems. ${ }^{7-16}$

Many types of PV cells used in modules are not perfectly square in shape. ${ }^{1,2}$ When assembled in modules, this results in a certain percentage of the module area not occupied by active PV cell material and solar illumination that is not collected for electrical conversion. Other factors, such as bus and finger contacts [exclude interdigitated back contact (IBC) cells] and the module package configuration, also contribute to inactive module surface area (IMSA) that does not contribute to PV solar energy conversion. Even the new half-cell modules designed to minimize the space between cells still have an IMSA ratio of $\sim 6 \%$. Table 1 shows several commercial solar panels' specifications with the IMSA ratio varying from $6 \%$ (half-cell module) to $11 \%$ (old module). The IMSA ratio is defined by IMSAR $=A_{\mathrm{HLC}} / A_{M} . A_{\mathrm{HLC}}$ and $A_{M}$ are the IMSA area and the whole PV module surface area, respectively.

In this paper, a light management technique to capture the solar energy falling on the IMSA is described using a holographic light collector (HLC) consisting of a reflection type volume

*Address all correspondence to Jianbo Zhao, jianbozhao@email.arizona.edu 
Zhao, Chrysler, and Kostuk: Holographic low concentration optical system increasing light collection...

Table 1 IMSA ratio of several commercial solar panels.

\begin{tabular}{llccc}
\hline \hline Company & \multicolumn{1}{c}{ Cell type } & $\begin{array}{c}\text { No. of } \\
\text { cells }\end{array}$ & $\begin{array}{c}\text { Front glass } \\
(\mathrm{mm})\end{array}$ & $\begin{array}{c}\text { IMSA } \\
(\%)\end{array}$ \\
\hline Jinko & Eagle HC 70M 365 to $385 \mathrm{~W}$ & 72 & 3.2 & 11.11 \\
Jinko & Eagle EP 60 280 to $300 \mathrm{~W}$ & 60 & 3.2 & 10.79 \\
Jinko & Eagle 72HM G5B 515 to 535 W & $2 \times 72$ & 3.2 & 6.60 \\
Trina solar & Tallmax Plus framed 60-cell module & 60 & 3.2 & 9.93 \\
Trina solar & Tallmax Plus framed 72-cell module & 72 & 3.2 & 9.01 \\
Trina solar & Tallmax Plus framed 144 half-cell module & $2 \times 72$ & 3.2 & 7.80 \\
\hline \hline
\end{tabular}

holographic element combined with a diffuser. The system is analyzed to determine the additional EY in a specific location (Tucson, Arizona) by considering the solar illumination's varied incident angle at different times and seasons. The results show that $53 \%$ of the illumination falling onto the IMSA can be collected and converted into extra electricity when two-axis tracking systems are deployed together with the PV systems. This corresponds to an increase in EY of $4.5 \%$ when the IMSA is $8.25 \%$ of the entire PV panel surface area. In addition, EY improvements of $4.1 \%$ and $3.8 \%$ can be realized, respectively, with single-axis tracking and fixed solar arrays. In Sec. 2, the system design aspects, including the geometrical configurations, PV cell spectral responsivity (SR), and HOE properties, are discussed. The power collection efficiency of the HLC is evaluated as a function of Sun angle changes during a day at different seasons, and the resulting improvement in EY is presented in Sec. 3. The results for an experimental HOE are presented in Sec. 4. Section 5 summarizes the performance of the HLC and its potential to increase the power collection efficiency of regular PV systems.

\section{System Design}

\subsection{Design Configuration}

Solar illumination falling onto the IMSA can be redirected onto the PV cells either by a transmission or reflection $\mathrm{HOE}^{7}$ An advantage of a reflective type HOE is that it can be encapsulated into existing PV module packages without deploying another cover glass needed by the transmission HOE. ${ }^{17}$ Also, light diffracted from an HOE can be designed to exceed the critical angle of the module's glass cover (Fig. 1). This effectively extends the distance over which light can be collected and transferred to the module's PV cell regions.

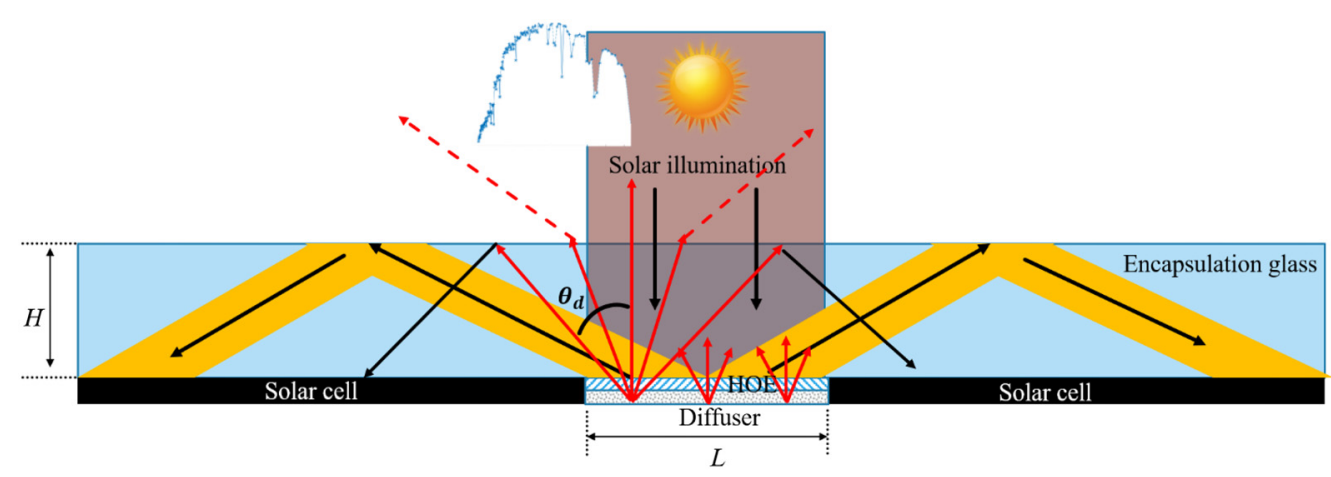

Fig. 1 System layout of the HLC. 
A simplified diagram of the HLC is shown in Fig. 1. The thickness of the solar panel's encapsulation glass is $H$, the width of the IMSA is $L$, and the refractive index of the glass cover is assumed to be 1.50. Holographic elements have both angular and spectral selectivity; therefore, as the angle and wavelength differ from the design values, the diffraction efficiency will decrease. A tracking system will keep the hologram at the design values resulting in a high collection efficiency. In contrast, a Lambertian diffuser has a relatively low sensitivity to the angle and wavelength variation of the incident solar illumination. Therefore, combining a holographic element with a diffuser can offer advantages for collecting solar illumination. In Fig. 1, the IMSA is covered with an HLC consisting of a reflection HOE constructed with wavelength selectivity matched to the PV cell's SR and angles and a Lambertian diffuser surface. For this configuration, the solar illumination incident on the HOE surface is partly diffracted into an angle that is larger than the critical angle of the glass cover. The diffuser scatters the remaining light that is not diffracted by the HOE. Some of this scattered light exceeding the cover glass's critical angle is reflected onto the PV cell surface and converted into electrical power and energy. Multiple interactions of light scattered by the diffuser and diffracted by the hologram that make it to the PV cell surface are also considered in the simulation.

The HOE is designed to be symmetric about the center of the IMSA to increase the length of the effective light collection region. The left half of the HOE diffracts light to a PV cell on the left side of the IMSA, and the right half of the HOE diffracts light to a cell on the right. The effective length describing the propagation distance of the diffracted/diffused light in the horizontal direction is given by

$$
l_{\text {eff }}=4 \cdot H \cdot \sin \left(\theta_{d}\right),
$$

where

$$
\theta_{d} \geq \theta_{\mathrm{CA}}=\sin ^{-1}\left(1 / n_{g}\right)
$$

$\theta_{d}$ is the diffraction angle and $H$ is the thickness of the encapsulation glass. The glass cover functions as a light waveguide to redirect light with angles larger than the glass cover's critical angle onto the cells. $n_{g}$ is the refractive index of the glass. $\theta_{\mathrm{CA}}$ is the total internal reflection angle of the cover, which is around $41.8 \mathrm{deg}$ for a glass with a refractive index of 1.50. Equation (1) indicates that the effective light collection area's length is proportional to the cover glass's thickness and the sinusoidal value of the redirected light's angle. For the commercial PV panels shown in Table 1, the shortest effective collection length is $8.53 \mathrm{~mm}$ when the thickness of the cover glass is $3.2 \mathrm{~mm}$. This means part of the diffracted light can interact with the HLC a second time when the length of the IMSA is longer than $8.53 \mathrm{~mm}$.

\subsection{Current Responsivity of Silicon Cells}

The SR characteristics vary significantly with different combinations of semiconductor materials such as GaAs, CIGS, and CdTe. ${ }^{1,2}$ To determine the appropriate peak wavelength of the HOE, the specific current responsivity (CR) of the PV cell needs to be evaluated by taking the product of the solar spectrum and the SR of the PV cells. The expression of the CR for a PV cell under the illumination with global standard AM1.5 spectrum is given by

$$
\mathrm{CR}(\lambda)=E_{\mathrm{AM} 1.5}(\lambda) \cdot \mathrm{SR}(\lambda) .
$$

In this paper, the monocrystalline silicon cell is used as the target material for the HLC design. The standard AM1.5G spectrum is depicted in Fig. 2(a), and the SR of the silicon cells is shown in Fig. 2(b). The CR of a monocrystalline silicon PV cell is shown in Fig. 2(c) when illuminated with AM1.5G spectrum. It implies that the optimal spectral range for HOE design ranges from 600 to $800 \mathrm{~nm}$. With proper multiplexing, index modulation profile, and thickness, an HOE can be designed to have a high DE with $>300 \mathrm{~nm}$ of spectral bandwidth. A function for optimizing the spectral bandwidth of the HOE is given by 
Zhao, Chrysler, and Kostuk: Holographic low concentration optical system increasing light collection...

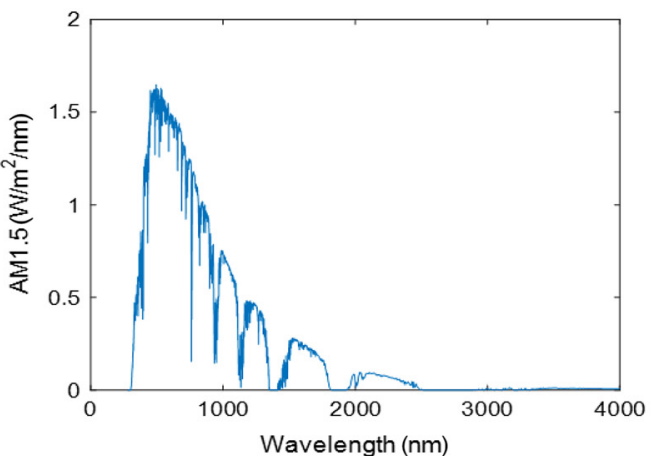

(a)

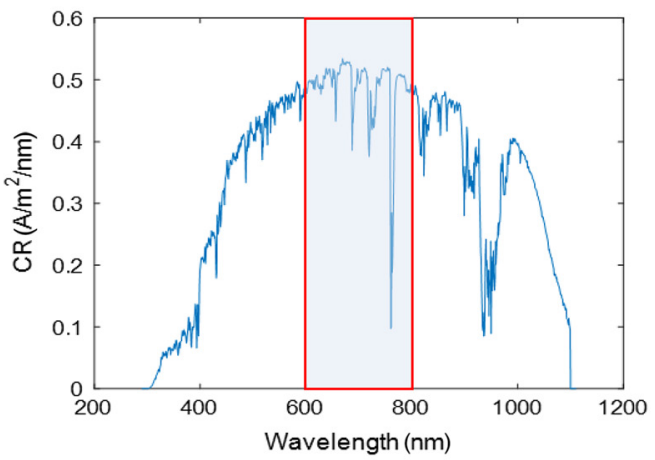

(c)

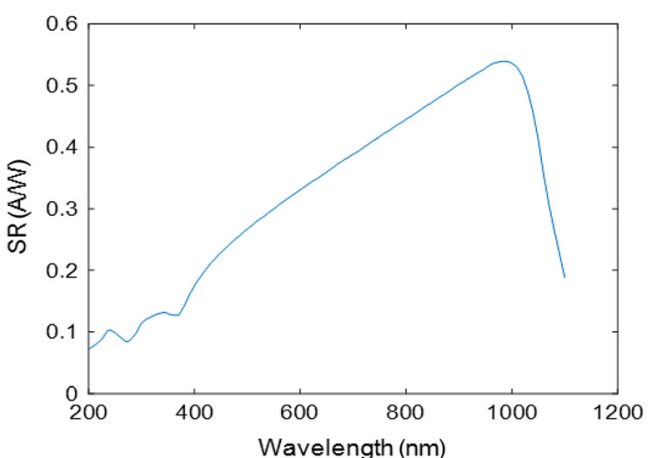

(b)

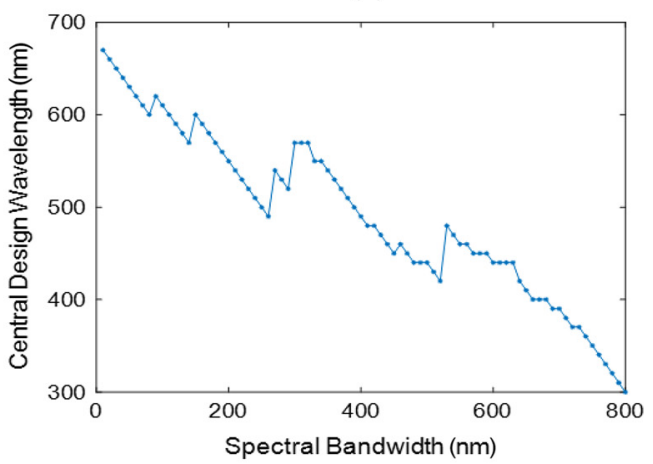

(d)

Fig. 2 (a) AM1.5G spectra; (b) SR of the silicon cells; (c) CR of the monocrystalline silicon cells; and (d) optimal design wavelength for the HOE as the function of the HOE's spectral bandwidth.

$$
\Gamma=\int_{\lambda_{\min }}^{\lambda_{\max }} \mathrm{CR}(\lambda) \cdot \operatorname{DE}(\lambda) \cdot \mathrm{d} \lambda,
$$

where $\operatorname{DE}(\lambda)$ is the diffraction efficiency of the HOE as the function wavelength in air. $\lambda_{\min }$ and $\lambda_{\max }$ are the minimum and maximum effective wavelength of the CR. The optimal design wavelength then can be calculated based on Eq. (4).

To illustrate the change in the hologram's peak efficiency wavelength as a function of the DE spectral bandwidth, assume that the DE of the hologram has a rectangular characteristic with wavelength. Also assume that the HOE and PV cell are illuminated with an AM1.5G spectrum [Fig. 2(a)], the PV cell is silicon [Fig. 2(b)], and the DE is $100 \%$ within the spectral bandwidth, as shown in Fig. 2(c). These factors are then used to compute the optimal $\Gamma$ as a function of the spectral bandwidth of the hologram and determine the design wavelength. Figure 2(d) shows the optimal design central wavelength of the HOE as the function of the HOE's spectral bandwidth. The result indicates that the optimal design wavelength for the HOE should be shifted to a shorter wavelength as the spectral bandwidth of the hologram increases.

\subsection{HOE Design}

There are two basic steps designing an HOE. The first is to determine the angles and wavelength of the construction and reconstruction conditions using Bragg matching conditions. ${ }^{18-21}$ The expression of the Bragg matching condition is given by

$$
\overrightarrow{\mathbf{K}}=\overrightarrow{\mathbf{k}}_{1, \lambda_{1}}-\overrightarrow{\mathbf{k}}_{2, \lambda_{1}}=\overrightarrow{\mathbf{k}}_{1, \lambda_{2}}-\overrightarrow{\mathbf{k}}_{2, \lambda_{2}}
$$

where $\overrightarrow{\mathbf{k}}_{1}$ and $\overrightarrow{\mathbf{k}}_{2}$ are the propagation vectors of the incident light and the diffraction light. $\overrightarrow{\mathbf{K}}$ is the grating vector that is perpendicular to the interference fringes and has a magnitude of $2 \pi / \Lambda$, where $\Lambda$ is the grating period. $\lambda_{1}$ and $\lambda_{2}$ are the wavelengths of the construction and 


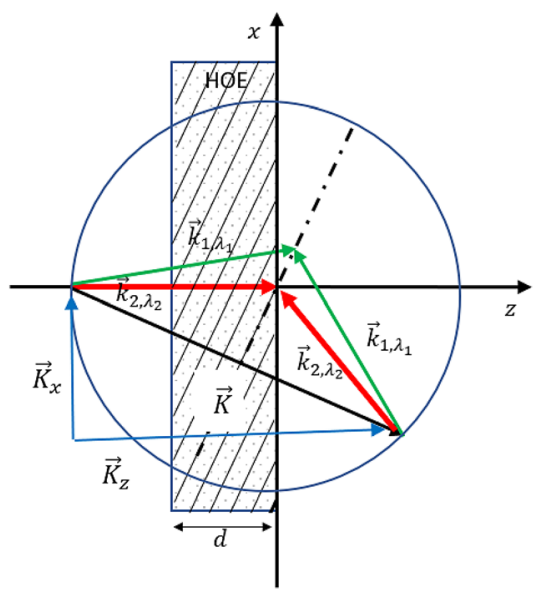

Fig. 3 Geometrical illustration of a reflection HOE with a thickness of $d$. Reconstruction conditions: $\lambda_{2}=600 \mathrm{~nm}, \theta_{1}=0.0 \mathrm{deg}$, and $\theta_{2}=135.0 \mathrm{deg}$. Construction conditions: $\lambda_{1}=532 \mathrm{~nm}$, $\theta_{1}=12.5 \mathrm{deg}$, and $\theta_{2}=122.5 \mathrm{deg} . \Lambda_{x}=558 \mathrm{~nm}$ and $\Lambda_{z}=231 \mathrm{~nm}$.

reconstruction beams, respectively. The second step in designing the hologram is to determine the index modulation $(\Delta n)$ and thickness $(d)$ to achieve the target DE requirements.

Using the methodology of Sec. 2.2, a reflection hologram with a 200-nm spectral bandwidth will have an optimum $\Gamma$ with a design wavelength of $600 \mathrm{~nm}$. A diagram of the Bragg condition for constructing a hologram at $\lambda_{1}$ and reconstructing it at $\lambda_{2}$ inside the holographic material is shown in Fig. 3, where $d$ is the thickness of the HOE. Based on the Bragg matching condition, the grating period in $x$ and $z$ directions can be derived as

$$
\Lambda_{x}=\frac{\lambda}{2 n_{h} \cos \left(\frac{\theta_{2}+\theta_{1}}{2}\right) \sin \left(\frac{\theta_{2}-\theta_{1}}{2}\right)}, \quad \Lambda_{z}=\frac{\lambda}{2 n_{h} \sin \left(\frac{\theta_{2}+\theta_{1}}{2}\right) \sin \left(\frac{\theta_{2}-\theta_{1}}{2}\right)},
$$

where $\theta_{1}$ and $\theta_{2}$ are the angles of the reference and object beams used for construction. All angles used for HOE design are relative to the $z$ direction and within the holographic material unless noted otherwise. $n_{h}$ is the refractive index of the HOE. $\lambda$ is the wavelength of the two construction beams. As depicted in Fig. 3, the reconstruction condition is set to $\lambda_{2}=600 \mathrm{~nm}$, $\theta_{1, \lambda 2}=0.0 \mathrm{deg}$, and $\theta_{2, \lambda 2}=135.0 \mathrm{deg}$ and the $\mathrm{HOE}$ is recorded at the wavelength $\lambda_{1}=$ $532 \mathrm{~nm}$ and angles $\theta_{1, \lambda 1}=12.5 \mathrm{deg}$ and $\theta_{2, \lambda 1}=122.5 \mathrm{deg}$.

For an unslanted reflection $\mathrm{HOE}$, the $\mathrm{DE}$ is given by

$$
\eta_{r}=\tanh ^{2}\left(\frac{\pi \Delta n d}{\lambda}\right)
$$

where $\Delta n$ is the index modulation, and $d$ is the thickness of the HOE. This expression indicates that the DE of a reflection HOE can be increased larger the thickness and index modulation; however, the diffraction efficiency starts to saturate.

One method to achieve a large spectral and angular bandwidth of the hologram is to vary the grating period as a function of depth within the hologram. ${ }^{15,22}$ It is possible to achieve this effect in recording materials such as dichromated gelatin. ${ }^{15-17,22}$ Figure 4(a) and 4(b) show the grating profiles in the $x-z$ plane within the hologram for the reflection hologram case described in Fig. 3. Figure 4(a) shows a uniform index modulation with depth, and Fig. 4(b) has a 20\% exponential swelling index profile with hologram depth. The maximum refractive index modulation for both cases is 0.08 , and the hologram thickness prior to the swelling process is $20 \mu \mathrm{m}$.

Figure 5 shows the diffraction efficiency as a function of wavelength and angle for the modulation profiles described in Figs. 4(a) and 4(b). The calculations were performed using rigorous coupled wave analysis, which allows consideration of the varied index modulation profile and the $x-y$ plane angle evaluations. As shown in Fig. 5(a), the spectral bandwidth of the hologram with swelling increases significantly (over $200 \mathrm{~nm}$ ) compared to the hologram with uniform 


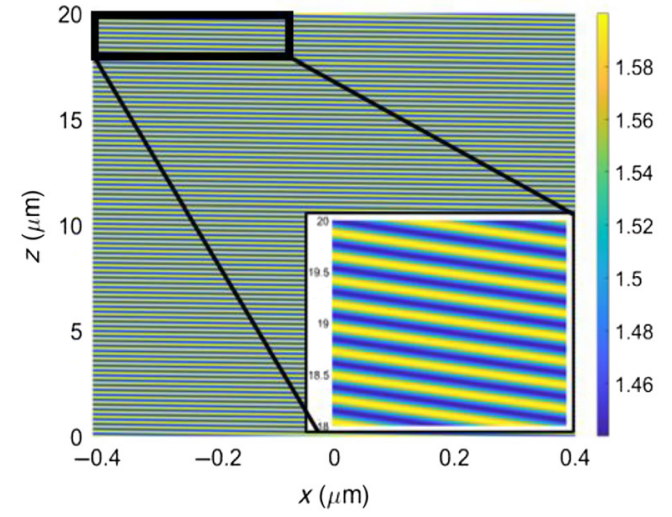

(a)

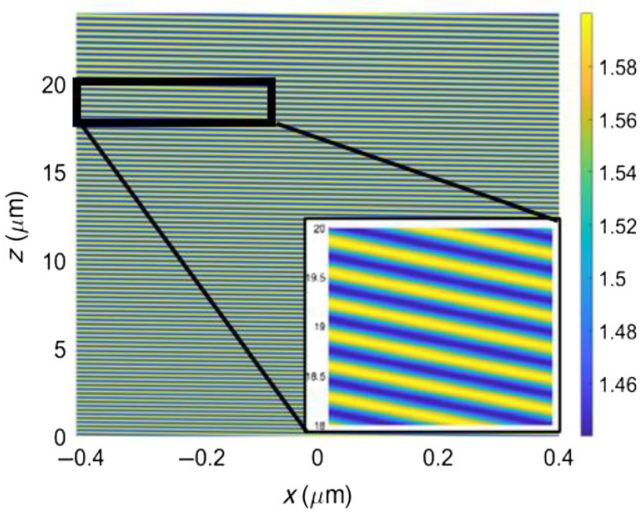

(b)

Fig. 4 (a) Grating profile of HOE in $x-z$ plane without swelling effects, where there are nine fringes between 18 and $20 \mathrm{~mm}$ in the thickness direction. The total thickness of the HOE is $d=20 \mu \mathrm{m}$. (b) HOE fringe profile in $x-z$ plane with a swelling coefficient $C=0.2$, with only six fringes between 18 and $20 \mathrm{~mm}$ in the thickness direction. The total thickness of the HOE is $d=24 \mu \mathrm{m}$ resulting from the swelling.

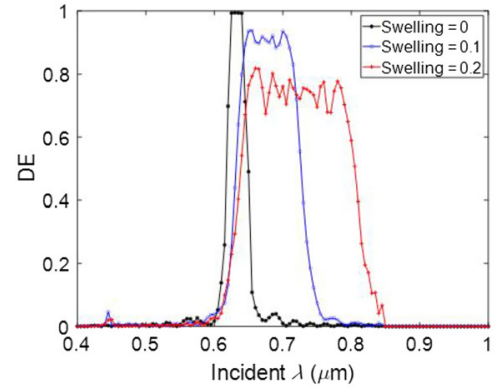

(a)

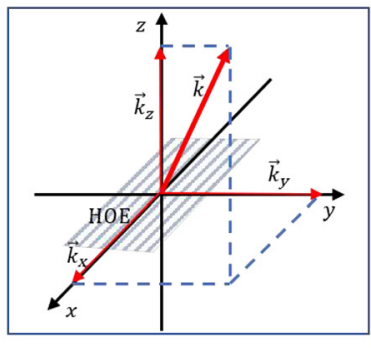

(b)

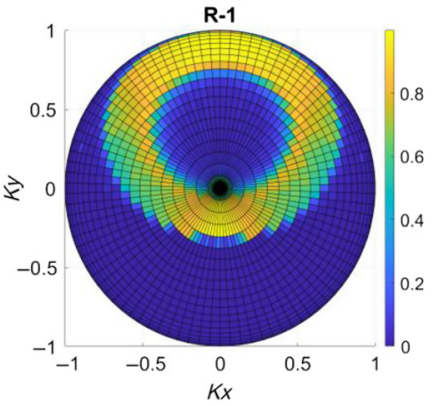

(c)

Fig. 5 (a) The spectral DE profiles of three HOEs with different swelling coefficients when the index modulation is fixed to 0.08 ; (b) the projected propagation vector components in $x, y$, and $z$ direction of the incident beam; and (c) the 3D angular DE profile in the first reflection order at $\lambda=600 \mathrm{~nm}$. The grating vector $\overrightarrow{\mathbf{K}}$ is along the $k_{y}$ direction.

refractive index modulation $(50 \mathrm{~nm})$ with the same prior-processing hologram thickness. The diffracted beam, in this case, exceeds the critical angle for wavelengths greater than the design wavelength. This allows the light to be guided to the location of PV cells and will increase the power collection efficiency and EY of the module.

The coordinates used for describing the incident beam are illustrated in Fig. 5(b). The normalized propagation vector of the incident beam is given by

$$
\vec{k}=\left[k_{x}, k_{y}, k_{z}\right]=[\sin (\theta) \cos (\phi), \sin (\theta) \sin (\phi), \cos (\theta)],
$$

where $k_{x}, k_{y}$, and $k_{z}$ are the normalized $x, y$, and $z$ components of the propagation vector. $\theta$ is the polar angle, and $\phi$ is the azimuthal angle in the spherical coordinates. The grating fringes are along the $k_{x}$ direction and the grating vector $\overrightarrow{\mathbf{K}}$ is along the $k_{y}$ direction. To accurately model the light collection efficiency of the HLC, a 3D angular DE map with the wavelength of incident beam varying from 300 to $1100 \mathrm{~nm}$ is generated, and Fig. 5(c) shows a specific 3D DE map in the first reflection order of the HOE when the wavelength of the reconstruction beam is $600 \mathrm{~nm}$ and the corresponding parameters used for calculation is presented in Table 2. As shown in Fig. 5(c), the angular DE map at a specific wavelength is donut-shaped in the 3D space. 
Zhao, Chrysler, and Kostuk: Holographic low concentration optical system increasing light collection...

Table 2 Parameters of HOE.

\begin{tabular}{lcc}
\hline \hline Parameter & Symbol & Value \\
\hline Thickness before swelling & $d$ & $20.0 \mu \mathrm{m}$ \\
Swelling coefficient & $C$ & 0.2 \\
Index modulation & $\Delta n$ & 0.08 \\
Construction wavelength & $\lambda_{1}$ & $532 \mathrm{~nm}$ \\
Reconstruction wavelength & $\lambda_{2}$ & $600 \mathrm{~nm}$ \\
Construction angles & $\left(\theta_{1, \lambda 1}, \theta_{2, \lambda 1}\right)$ & $(12.5 \mathrm{deg}, 122.5 \mathrm{deg})$ \\
Reconstruction angles & $\left(\theta_{1, \lambda 2}, \theta_{2, \lambda 2}\right)$ & $(0.0 \mathrm{deg}, 135.0 \mathrm{deg})$ \\
\hline \hline
\end{tabular}

\section{Energy Yield Evaluation}

\subsection{Incident Solar Illumination Angle}

The hologram diffraction efficiency and the EY are highly dependent on the angle of incident solar illumination relative to the normal of the hologram surface. The Sun's angle relative to a surface normal, in turn, depends on the geographical location, time of the day, and the season of the year. The zenith $\theta_{S, Z E}$ and azimuth $\varphi_{S, A Z}$ angles of the Sun can be readily obtained from wellknown Sun position algorithms. ${ }^{23}$ Sun position values can be combined with parameters for the geographical location and system deployment method (i.e., dual-axis tracking, single-axis tracking, or fixed angle arrays) to determine the angle of incidence and irradiance at the module surface. To simplify the EY calculation process for evaluating the hologram collection efficiency, the incident solar spectrum is assumed to be the AM1.5G spectrum with a total input irradiance of $1000 \mathrm{~W} / \mathrm{m}^{2}$.

The solar angle can be described by the elevation angle $\left(\theta_{S, \mathrm{EL}}=90 \mathrm{deg}-\theta_{S, \mathrm{ZE}}\right)$ and azimuth angle $\left(\theta_{S, \mathrm{AZ}}\right)$ that are given by the following equations:

$$
\begin{gathered}
\theta_{S, \mathrm{EL}}\left(t_{d}, t_{h}\right)=\sin ^{-1}\left\{\sin \left[\delta\left(t_{d}\right)\right] \sin (\phi)+\cos \left[\delta\left(t_{d}\right)\right] \cos (\phi) \cos \left[\mathrm{HRA}\left(t_{h}\right)\right]\right\} \\
\varphi_{S, \mathrm{AZ}}\left(t_{d}, \mathrm{t}_{h}\right)=\cos ^{-1}\left\{\frac{\sin [\cos (\phi)]-\cos \left[\delta\left(t_{d}\right)\right] \sin d(\phi) \cos \left[\mathrm{HRA}\left(t_{h}\right)\right]}{\cos \left(\theta_{S, \mathrm{EL}}\right)}\right\},
\end{gathered}
$$

where $\phi$ is the latitude angle, $t_{h}$ is the number of hours in a day, and $t_{d}$ is the number of days since the start of the year. $\delta$ is the declination angle defined by

$$
\sin (\delta)=\sin (-23.45 \mathrm{deg}) \cos \left[\frac{360}{365}\left(t_{d}+10\right)\right] .
$$

HRA is the hour angle that converts the local solar time (LST) into the angle that travels across the sky daily, and it is given by

$$
\operatorname{HRA}\left(t_{h}\right)=15 \operatorname{deg}\left[\operatorname{LST}\left(t_{h}\right)-12\right]
$$

With the Sun's elevation angle and azimuth angle, the track of the Sun can be depicted as a vector that is given by

$$
\overrightarrow{\mathbf{k}}_{\mathrm{solar}}=\left[\cos \left(\theta_{S, \mathrm{EL}}\right) \sin \left(\varphi_{S, \mathrm{AZ}}\right), \cos \left(\theta_{S, \mathrm{EL}}\right) \cos \left(\varphi_{S, \mathrm{AZ}}\right), \sin \left(\theta_{S, \mathrm{EL}}\right)\right] .
$$

Moreover, when the PV module's surface normal is not perpendicular to the ground surface, in other words, it has a zenith angle and azimuth angle described by $\left(\theta_{M, \mathrm{ZE}}, \varphi_{M, \mathrm{AZ}}\right)$, the angle of the solar illumination relative to the module's surface normal is then given by 
Zhao, Chrysler, and Kostuk: Holographic low concentration optical system increasing light collection...

$$
\gamma=\cos ^{-1}\left[\cos \left(\theta_{M, \mathrm{EL}}\right) \cos \left(\theta_{S, \mathrm{EL}}\right) \cos \left(\varphi_{M, \mathrm{AZ}}-\varphi_{S, \mathrm{AZ}}\right)+\sin \left(\theta_{M, \mathrm{EL}}\right) \sin \left(\theta_{S, \mathrm{EL}}\right)\right]
$$

where $\theta_{M \text {,EL }}$ is the elevation angle of the PV module's surface normal, and it is related to its zenith angle by $\theta_{M, \mathrm{EL}}=90 \mathrm{deg}-\theta_{M, \mathrm{ZE}}$.

\subsection{Performance Evaluation}

The HLC consisting of an HOE and a diffuser is in combination of the PV cells shown in Fig. 6. The PV cell has a dimension of $W_{\mathrm{PV}} \times W_{\mathrm{PV}}$, the reflectivity of the white diffuser is $R_{D}$, and the IMSA has a width of $L$. Figure 6(b) shows a single PV module where $A_{\mathrm{HLC}}$ and $A_{M}$ are the surface areas of the IMSA and PV cells, respectively, and they are given by

$$
A_{M}=\left(W_{\mathrm{PV}}+L\right)^{2}, \quad A_{\mathrm{HLC}}=2 \cdot\left(W_{\mathrm{PV}}+L\right) \cdot L-L^{2} / 2 .
$$

The electric power output of the PV cell without HLC is defined by

$$
\begin{gathered}
P_{\text {cell }}\left(t_{s}\right)=A_{M} \cdot \int_{\lambda_{\min }}^{\lambda_{\max }} E_{\mathrm{AM} 1.5}\left(\lambda, t_{s}\right) \cdot \mathrm{SR}(\lambda) \cdot \mathrm{FF} \cdot V_{\mathrm{OC}} \cdot \mathrm{d} \lambda \\
=A_{M} \cdot \mathrm{FF} \cdot V_{\mathrm{OC}} \cdot \int_{\lambda_{\text {min }}}^{\lambda_{\max }} E_{\mathrm{AM} 1.5}\left(\lambda, t_{s}\right) \cdot \mathrm{SR}(\lambda) \cdot \mathrm{d} \lambda .
\end{gathered}
$$

FF is the fill factor and $V_{\mathrm{OC}}$ is the open circuit voltage of the PV cells, and they are assumed to be constants over the operating condition and wavelength range. $t_{s}$ is the LST that determines the solar angle $\left(\theta_{S, \mathrm{EL}}, \varphi_{S, \mathrm{AZ}}\right) . \lambda_{\min }$ and $\lambda_{\max }$ are the shortest and longest wavelengths determined by the $\operatorname{SR}(\lambda)$ boundaries of PV cells. The electric power output contributed by the HLC is given by

$$
P_{\mathrm{HLC}}\left(t_{i}\right)=A_{\mathrm{HLC}} \cdot \mathrm{FF} \cdot V_{\mathrm{OC}} \cdot \int_{\lambda_{\min }}^{\lambda_{\max }} E_{\mathrm{AM} 1.5}\left(\lambda, t_{s}\right) \cdot \mathrm{SR}(\lambda) \cdot \mathrm{OCE}\left(\lambda, t_{s}\right) \cdot \mathrm{d} \lambda,
$$

where $\operatorname{OCE}\left(\lambda, t_{s}\right)$ is the optical collection efficiency of the HLC at a specific wavelength and time. In order to quantify the improvement in the module performance resulting from the HLC, a normalized collected power ratio (NCPR) of the HLC is defined as the ratio of the electric power recycled by the HLC to the electric power converted directly by the PV cells. It is given by

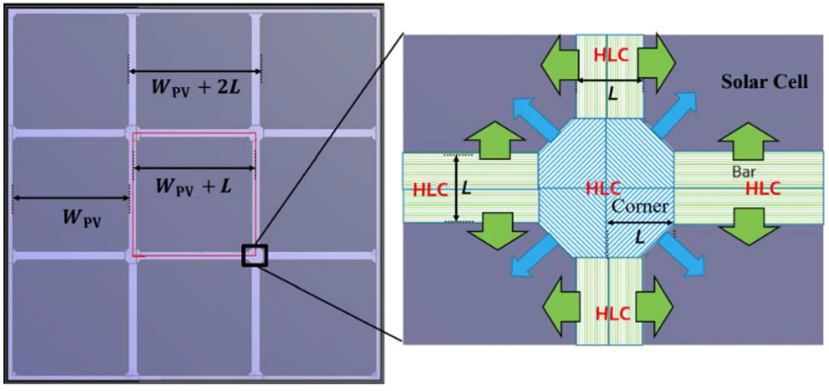

(a)

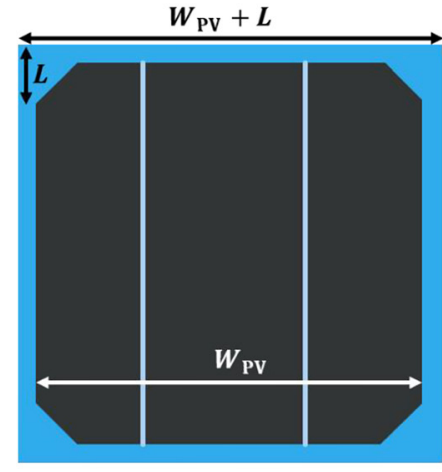

(b)

Fig. 6 (a) The left figure shows the diagram of the modeled system consisting of the HLC and PV cells; the light blue regions represent the IMSA; the dark blue regions are the PV cells that absorb all incident light with an efficiency of $100 \%$. The right figure is a zoomed region of the PV panel; the lines in the graph indicate the grating fringes direction; the green arrows show the effective light propagation directions after the reflection. (b) The zoomed figure showing the dimension of a single PV module. Note that for a standard PV module design, the horizontal and vertical widths of the IMSA are different. 
Zhao, Chrysler, and Kostuk: Holographic low concentration optical system increasing light collection...

$$
\operatorname{NCPR}\left(t_{s}\right)=\frac{P_{\mathrm{HLC}}\left(t_{s}\right)}{P_{\text {cell }}\left(t_{s}\right)}=\frac{A_{\mathrm{HLC}}}{A_{M}-A_{\mathrm{HLC}}} \frac{\int_{\lambda_{\min }}^{\lambda_{\max }} E_{\mathrm{AM} 1.5}\left(\lambda, t_{s}\right) \cdot \operatorname{SR}(\lambda) \cdot \mathrm{OCE}\left(\lambda, t_{s}\right) \cdot \mathrm{d} \lambda}{\int_{\lambda_{\min }}^{\lambda_{\max }} E_{\mathrm{AM} 1.5}\left(\lambda, t_{s}\right) \cdot \mathrm{SR}(\lambda) \cdot \mathrm{d} \lambda} .
$$

The annual energy yield improvement (AEYI) can be obtained by taking the ratio of the EY of HLC to the EY of regular PV cells without HLC and is given by the following equation:

$$
\mathrm{AEYI}=\frac{\mathrm{EY}_{\mathrm{HLC}}}{\mathrm{EY}_{\text {cell }}}=\frac{\sum P_{\mathrm{HLC}}\left(t_{s}\right) \cdot \Delta t}{\sum P_{\text {cell }}\left(t_{s}\right) \cdot \Delta t}=\frac{\sum P_{\mathrm{HLC}}\left(t_{s}\right)}{\sum P_{\text {cell }}\left(t_{s}\right)},
$$

where $\Delta t$ is the time interval between the adjacent solar times used for computation.

\subsection{Influence of Thickness of the Cover Glass}

Equation (1) indicates that the effective light collection length $l$ is proportional to the thickness of the encapsulation glass $(H)$ (i.e., a thicker cover glass results in a longer collection length). The NCPR performance as a function of the cover glass thickness $(H)$ for an HLC consists only a diffuser and one with only an HOE. The illumination considered for this aspect is on the IMSA only. Table 3 gives the other parameters used for calculation. Figure 7(a) illustrated the distribution of the rays absorbed by the PV cells when light is normally incident on the IMSA, and it indicates that most rays are absorbed near the edges of PV cells.

The NCPR for the HOE-only and the diffuser-only cases as a function of the thickness of the cover glass are shown in Fig. 7(b). As shown, NCPR increases for both cases when the cover

Table 3 Specifications used for the simulation.

\begin{tabular}{lcc}
\hline \hline Parameter & Symbol & Value \\
\hline PV cell dimension & $W_{\mathrm{PV}}$ & $160.0 \mathrm{~mm}$ \\
Width of IMSA & $L$ & $6.4 \mathrm{~mm}$ \\
Diffuser reflectivity & $R_{D}$ & $96.0 \%$ \\
Thickness of cover glass & $H$ & $3.2 \mathrm{~mm}$ \\
Inactive surface area ratio & IMSAR & $8.25 \%$ \\
\hline \hline
\end{tabular}

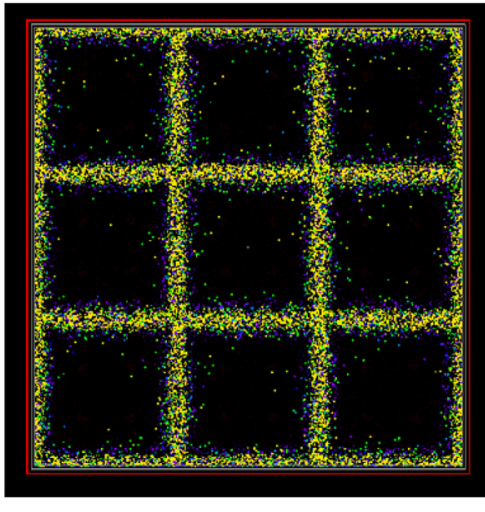

(a)

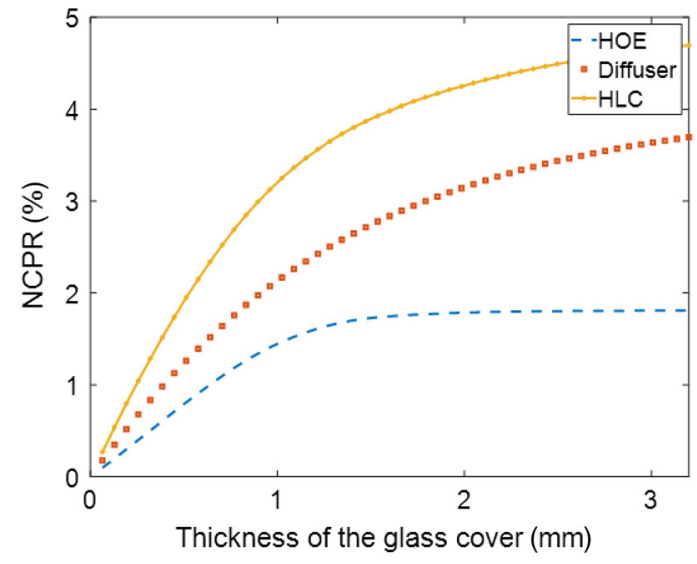

(b)

Fig. 7 (a) Irradiance distribution over the detector (PV cells). (b) NCPR as the function of the thickness of the glass cover for the HOE-only case, scatter-only case, and the HLC case when the IMSA's width is fixed to $6.4 \mathrm{~mm}$ and light incident normally. 
Zhao, Chrysler, and Kostuk: Holographic low concentration optical system increasing light collection...

glass gets thicker. The NCPR of the diffuser-only case is higher than the NCPR of the HOE-only case due to its broader spectral bandwidth; however, the NCPR of the HOE-only case approaches the saturation point much faster than the NCPR of the diffuser-only case resulting from the HOE's higher angle selectivity. Thus for a solar panel with a thin $(<3.2 \mathrm{~mm})$ encapsulation glass, the combination of the HOE and diffuser can provide a better light redirecting than the HOE-only or diffuser-only case. Although a higher NCPR is expected when a thicker glass cover is used, the cover glass thickness is chosen to be $3.2 \mathrm{~mm}$ since this corresponds to the value for most commercial PV modules (Table 1).

\subsection{AEYI for Dual-Axis and Single-Axis Tracking Systems with HLC}

According to Sec. 3.1, the Sun's angle at a specific location can be calculated using the corresponding latitude and longitude coordinates and the local time and season values. For this example, the coordinates $\left(32.2226^{\circ} \mathrm{N}, 110.9747^{\circ} \mathrm{W}\right)$ for Tucson, Arizona, are used. The solar angle is then expressed in the k-space described by $k_{x}$ and $k_{y}$ given in Eq. (13). The east-west direction corresponds to the $k_{x}$ direction and the south-north direction corresponds to the $k_{y}$ direction. A dual-axis tracking system can dynamically track the Sun's position and always ensure $k_{x}=k_{y}=0$ to maintain the highest light collection efficiency. When employed with a dual-axis tracking system, the HLC keeps its highest NCPR (4.5\%) at all times and seasons, resulting in an AEYI of $4.5 \%$ when the PV module has an IMSAR of $8.25 \%$. This implies that for a system like that described in Table 3 which generates $100 \mathrm{kWh} / \mathrm{year}$, an extra $4.5 \mathrm{kWh} /$ year can be achieved when an HLC and a dual-axis-tracking are used.

Single-axis tracking systems are less expensive but do not compensate for seasonal changes in the Sun angle. Figure 8 shows the variation in solar angle in Tucson, Arizona, as a function of $k_{x}$ and $k_{y}$. In this case, with the conditions given in Table 3, the NCPR has a maximum value of $4.5 \%$ when $k_{x}=k_{y}=0$ (normal incident), and the corresponding AEYI is $4.1 \%$. This implies that for a system like that described in Table 3 which generates $100 \mathrm{kWh} /$ year, an extra $4.1 \mathrm{kWh} /$ year can be achieved when an HLC and single-axis tracking are used.

\subsection{AEYI for Fixed Arrays with HLC}

Finally, considering a fixed array PV system with HLC module parameters as given in Table 3, if it is deployed in Tucson at a tilt angle of $32 \mathrm{deg}$, the highest NCPR is again $4.5 \%$ when the solar illumination is normally incident $\left(k_{x}=k_{y}=0\right)$ and decreases for other Sun angles (shown in

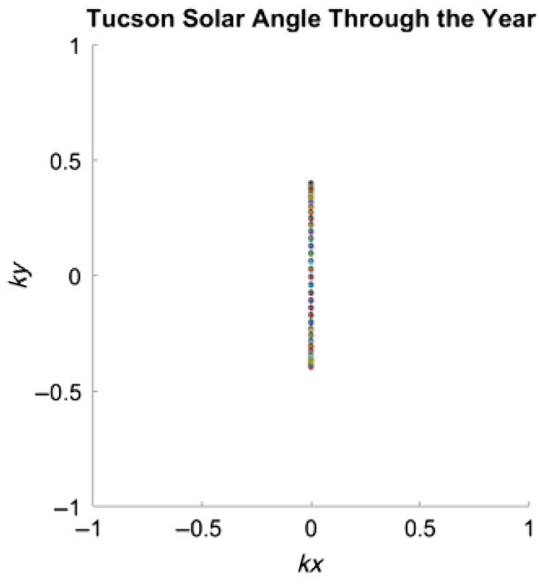

(a)

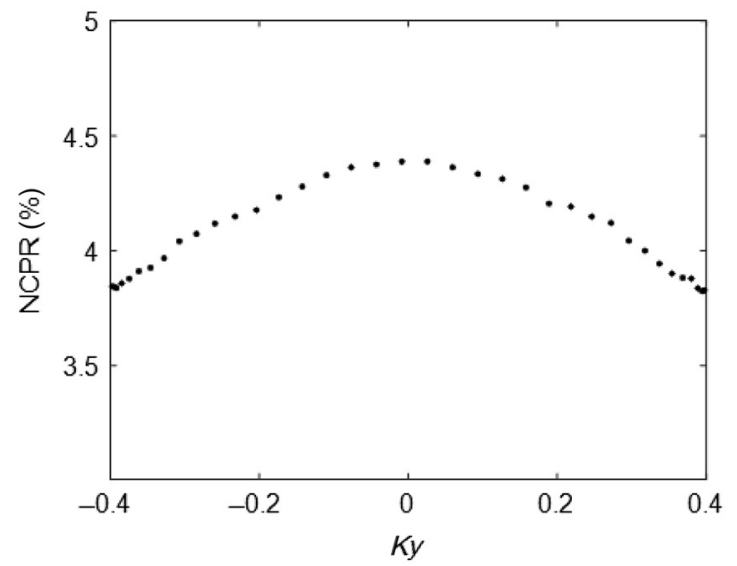

(b)

Fig. 8 (a) The solar angle varies as a function of specific times throughout the year in Tucson when the PV panels are deployed with single-axis tracking systems. (b) The NCPR of the HLC changes as the function of the solar angle at specific times throughout the year. 


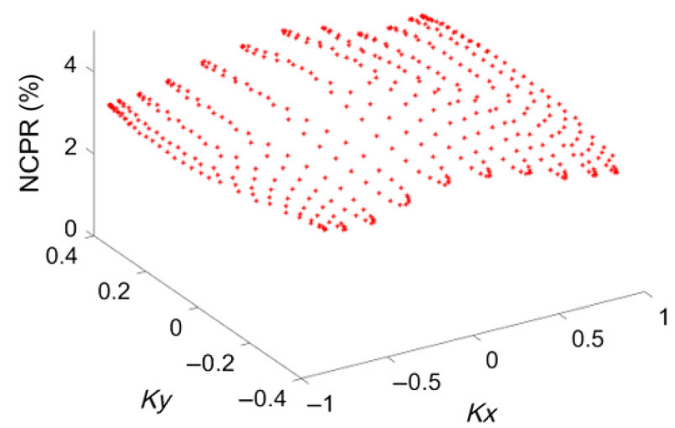

(a)

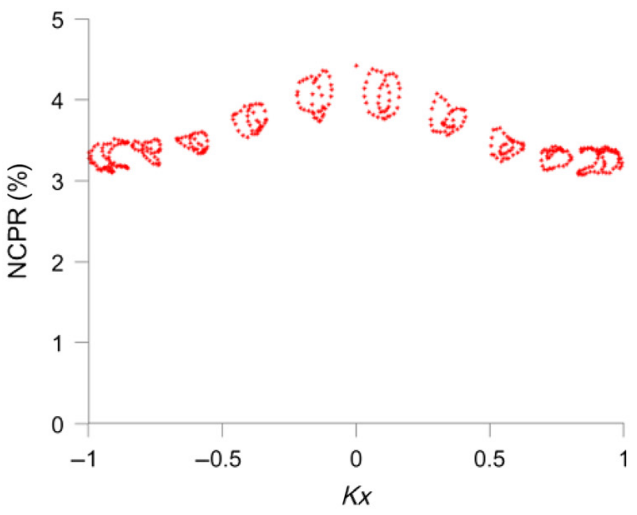

(b)

Fig. 9 The NCPR varies as the function of solar angle in (a) 3D space and (b) the plane of $k_{y}=0$.

Table 4 AEYI of the HLC employed with different systems.

\begin{tabular}{lccc}
\hline \hline Illumination type & $\begin{array}{c}\text { Fixed } \\
\text { arrays (\%) }\end{array}$ & $\begin{array}{c}\text { Single-axis } \\
\text { tracking (\%) }\end{array}$ & $\begin{array}{c}\text { Dual-axis } \\
\text { tracking (\%) }\end{array}$ \\
\hline Direct illumination & 3.8 & 4.1 & 4.5 \\
Direct + diffuse (20\%) & 3.6 & 4.0 & 4.3 \\
\hline \hline
\end{tabular}

Fig. 9). This gives an AEYI of 3.8\% and implies an extra $3.8 \mathrm{kWh} /$ year can be attained when an HLC is used with a fixed angle PV module array, compared to the same fixed module array without an HLC that produces $100 \mathrm{kWh} /$ year. This AEYI is very close to the diffuser-only case with an NCPR of 3.5\% [see Fig. 7(b)] since the holographic element contributes less to the total power collection efficiency while the incident angle deviates from the PV module's surface normal significantly. Thus a dual-tracking system is needed to make the HLC achieve the highest collection efficiency.

The AEYIs of the HLC deployed with fixed arrays, single-axis tracking, and dual-axis tracking systems are summarized in Table 4, where the AEYIs considering the diffuse light that contributes to $20 \%$ of the total illumination are also given.

\section{Experimental Results}

In order to verify some of the simulation results, experimental reflection holograms were formed in a commercial photopolymer recording material (Covestro HF200). This material has a thickness of $16 \mu \mathrm{m}$ and a maximum refractive index modulation of 0.03 . Reflection holograms were designed using the method described in Sec. 2.3. The experimental setup is shown in Fig. 10 for forming the hologram with light from a 532-nm laser and maximum diffraction efficiency at normal incidence with a reconstruction wavelength of $600 \mathrm{~nm}$. The reconstruction angles are determined as $0 \mathrm{deg}$ and $135 \mathrm{deg}$ and the corresponding construction angles are $12.5 \mathrm{deg}$ and $122.5 \mathrm{deg}$, and this results in a maximum diffraction efficiency at $600 \mathrm{~nm}$. The holographic photopolymer is sandwiched between two right-angle prisms used for in- and out-coupling the two construction beams.

The spectral diffraction efficiency of the recorded HOE is measured using an Ocean Optics USB 2000 spectrometer and indicates that the peak diffraction efficiency occurs at a wavelength of $600 \mathrm{~nm}$. Figure 11 shows a plot of the measured and theoretical diffraction efficiency as a function of the reconstruction wavelength computed using ACWA. It indicates a shift of the spectra when the angle of incident illumination varies. The comparison has a best-fit refractive index modulation of 0.017 . The upwelling of the sidelobes is an indication of the low collimation degree of the broadband testing illumination. In this case, the spectral bandwidth of the HOE is 


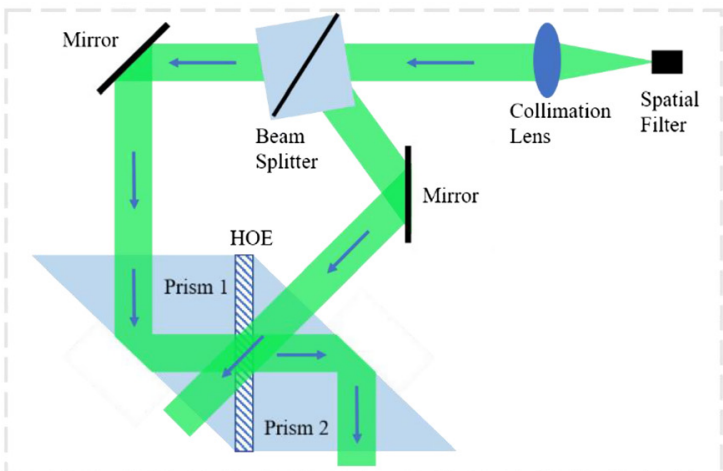

(a)

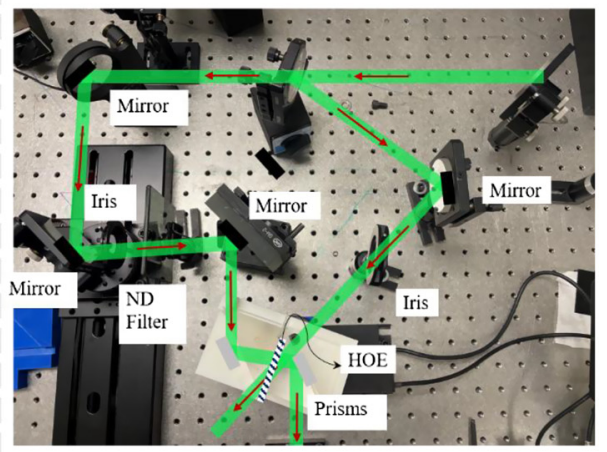

(b)

Fig. 10 (a) Configuration of the HOE recording setup and (b) the experimental setup used for recording HOE.

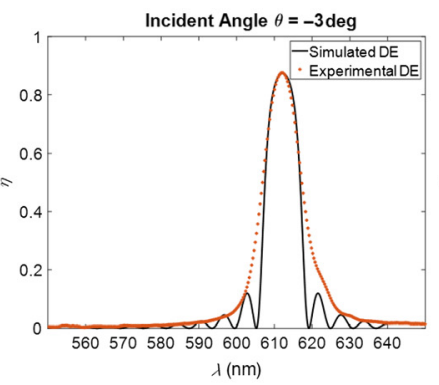

(a)

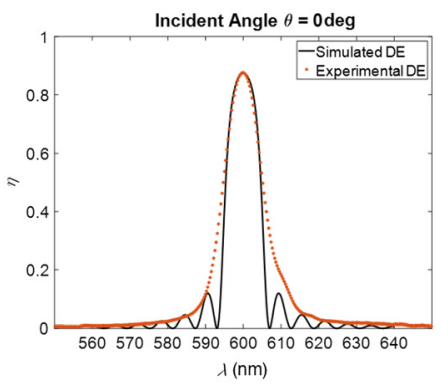

(b)

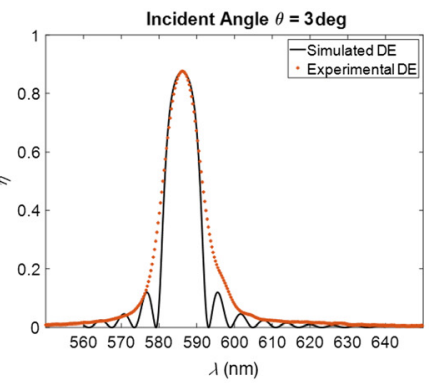

(c)

Fig. 11 The HOE's diffraction efficiency in the first reflection order as a function of reconstruction wavelength when the incident light has angles of (a) $-3 \mathrm{deg}$, (b $0 \mathrm{deg}$, and (c) $3 \mathrm{deg}$.

limited to $14 \mathrm{~nm}$ resulting from a low-index modulation amplitude, and the corresponding HLC's AEYI is decreased to $3.7 \%$ when this HOE is combined into the HLC and deployed with a PV system specified in Table 3 (with dual-axis tracking). It has been shown that with the proper recording methods and warm processing techniques, the spectral bandwidth of the HOE made in DCG ranges from 10 to $350 \mathrm{~nm} .^{22}$ For an HOE formed in dichromated gelatin with the parameters given in Table 2 (with a prior-processing thickness of $20 \mathrm{~m}$, an index modulation of 0.08 , and an exponentially swelling coefficient of 0.2 ), the spectral bandwidth can approach $250 \mathrm{~nm}$ [Fig. 5(a)] and provide an AEYI of $4.5 \%$ when it is combined into the HLC and PV systems with parameters given in Table 3.

\section{Conclusions}

In this paper, an HLC system consisting of a holographic optical element and a diffuser used to capture solar illumination falling onto the IMSA is proposed and analyzed. HLC configurations are formulated in the FRED optical simulation program and used to evaluate the power collection efficiency and AEYI. The annual EY analysis is computed for Tucson, Arizona, with dual-axis and single-axis tracking and a fixed non-tracking PV system. The corresponding improvements to the annual EY for a system with modules that have an IMSA of $8.25 \%$ are, respectively, $4.5 \%$, $4.1 \%$, and $3.8 \%$ with direct illumination. With direct and diffuse solar illumination, the corresponding AEYIs are, respectively, 4.3\%, 4.0\%, and 3.6\%. Notice that the IMSA ratio of a commercial module might be smaller than the value used in this analysis, resulting in a smaller gain. The IMSA distribution has been assumed to be uniform for simplification purposes, but it is not uniformly distributed for standard modules. Future work will focus on the experimental 
Zhao, Chrysler, and Kostuk: Holographic low concentration optical system increasing light collection...

evaluation of HLCs formed in materials with optimized thickness and refractive index modulation profiles such as dichromated gelatin.

\section{Acknowledgments}

This material is based upon work primarily supported by the National Science Foundation (NSF) and Department of Energy (DOE) funded QESST Engineering Research Center (No. EEC1041895). Any opinions, findings, and conclusions or recommendations expressed in this material are those of the author(s) and do not necessarily reflect those of the NSF or DOE.

\section{References}

1. M. A. Woodhouse et al., "Crystalline silicon photovoltaic module manufacturing costs and sustainable pricing: 1H 2018 Benchmark and Cost Reduction Road Map," National Renewable Energy Lab.(NREL), Golden, Colorado (2019).

2. E. Lorenzo, "Energy collected and delivered by PV modules," in Handbook of Photovoltaic Science and Engineering, A. Luque and S. Hegedus, Eds., pp. 906-930, Wiley (2002).

3. National Renewable Energy Laboratory, Best Research-Cell Efficiency Chart. https://www .nrel.gov/pv/cell-efficiency.html (accessed 4 April 2021).

4. National Renewable Energy Laboratory, Best Research-Cell Efficiency Chart. https://www .nrel.gov/pv/module-efficiency.html (accessed 4 April 2021).

5. F. Schindler et al., "Towards the efficiency limits of multicrystalline silicon solar cells," Solar Energy Mater. Solar Cells 185, 198-204 (2018).

6. A. Luque, Solar Cells and Optics for Photovoltaic Concentration, pp. 305, 355, Institute of Physics (1989).

7. J. E. Ludman, "Holographic solar concentrator" Appl. Opt. 21(17), 3057-3058 (1982).

8. Y. W. Zhang et al., "Photovoltaic concentrator using a holographic optical element," Appl. Opt. 27(16), 3556-3560 (1988).

9. C. Bainier, C. Hernandez, and D. Courjon, "Solar concentrating systems using holographic lenses," Solar Wind Technol. 5, 395-404 (1988).

10. J. Ludman and J. Riccobon, "Holographic solar concentrator for terrestrial photovoltaics," in Conf. Rec. of the 1994 IEEE First World Conf. Photovoltaic Energy Convers, IEEE (1994).

11. R. K. Kostuk, J. Castro, and D. Zhang, "Holographic low concentration ratio solar concentrators," in Front. Opt., Optical Society of America (2009).

12. D. Zhang et al., "Ultra light-trapping filters with broadband reflection holograms," Opt. Express 20(13),14260-14271 (2012).

13. J. Zhao et al., "Improved solar illumination collection using holographic light management techniques," Proc. SPIE 11120, 111200L (2019).

14. A. G. Imenes and D. R. Mills, "Spectral beam splitting technology for increased conversion efficiency in solar concentrating systems: a review," Solar Energy Mater. Solar Cells 84(1-4), 19-69 (2004).

15. J. M. Russo et al., "Spectrum splitting metrics and effect of filter characteristics on photovoltaic system performance," Opt. Express, 22(S2), A528-A541 (2014).

16. B. D. Chrysler et al., "Spectrum-splitting photovoltaic system using bifacial cells for high energy yield," Proc. SPIE 11121, 111210B (2019).

17. J. Russo et al., "Characterization of diffuse anisotropic illumination effects to the output of bifacial and holographic planar concentrating photovoltaic panel configurations," in 38th IEEE Photovoltaic Specialists Conf. (2012).

18. C. G. Stojanoff, "Review of the technology for the manufacturing of large-format DCG holograms for technical applications," Proc. SPIE 3011, 267-278 (1997).

19. J. Goodman, Introduction to Fourier Optics, pp. 297-393, McGraw-Hill (1998).

20. H. Kogelnik, "Coupled wave theory for thick hologram gratings," Bell Syst. Technol. J. 48, 2909-2947 (1969). 
Zhao, Chrysler, and Kostuk: Holographic low concentration optical system increasing light collection...

21. M. G. Moharam and T. K. Gaylord, "Three-dimensional vector coupled-wave analysis of planar-grating diffraction,” J. Opt. Soc. Am. 73, 1105-1112 (1983).

22. L. Solymar and D. J. Cooke, Volume Holography and Volume Gratings, Academic (1981).

23. I. Reda and A. Andreas, "SPA: solar position algorithm," Astrophysics Source Code Library, Ascl:1504.002 (2015).

Jianbo Zhao received his BS degree in physics from Nankai University and his MS degree in optics from James C. Wyant College of Optical Sciences in 2015 and 2018. He is a PhD student at the University of Arizona. He is working on improving solar and AR systems performance by designing volume holographic elements under the supervision of Dr. Raymond K. Kostuk.

Biographies of the other authors are not available. 